\title{
Stokeslet and the Operator Extensions Theory
}

\author{
I.YU, POPOV
}

\begin{abstract}
Operator version of the stokeslet method in the theory of creeping flow is suggested. The approach is analogous to the zero-range potential one in quantum mechanics and is based on the theory of self-adjoint operator extensions in the space $L_{2}$ and in the Pontryagin's space with an indefinite metric. The problem of Stokes flow in two channels connected through a small openings is considered in the framework of this approach. The case of a periodic system of small openings is studied too. The picture of streamlines for such flow is obtained.
\end{abstract}

\section{INTRODUCTION}

The use of singular solutions of Stokes equations is extremely useful in the theory of creeping flow (low-Reynolds-number flow). Let us consider the Stokes equations

$$
\begin{gathered}
\nabla \cdot \vec{v}=0 \\
-\nabla p+\mu \Delta \vec{v}=-\vec{A} \delta\left(\vec{x}-\vec{x}_{0}\right) .
\end{gathered}
$$

1991 Mathematics Subject Classification: 47F, 76D

Servicio publicaciones Univ. Complutense. Madrid, 1996. 
Here $\vec{v}\left(v_{i}\right), p, \mu$ are a velocity, pressure and viscosity correspondingly. The solution $p_{s}, \vec{v}_{s}$ :

$$
\begin{aligned}
& p_{s}=(\vec{A} \cdot \nabla) \varphi_{s}, \\
& \mu \vec{v}=\frac{1}{2} \nabla\left(\vec{x} \cdot \vec{A} \varphi_{s}\right)-\vec{A} \varphi_{s}-\frac{1}{2}\left(\vec{A} \cdot \vec{x}_{0}\right) \nabla \varphi_{s},
\end{aligned}
$$

where

$$
\varphi_{s}= \begin{cases}-\left(4 \pi\left|\vec{x}-\vec{x}_{0}\right|\right)^{-1} & \text { in } 3-\text { dimensional case } \\ -(2 \pi)^{-1} \ln \left|\vec{x}-\vec{x}_{0}\right| & \text { in } 2-\text { dimensional case }\end{cases}
$$

of the equations (1) is called a "stokeslet" [1]. One can use these solutions for study of the Stokes flow near some bodies [2], for the investigation of the self-propulsion of microscopic organism through liquids [1], for the research of interesting examples of creeping flows in a pipe or between plates [3 - 5] and for many other purposes $[6,7]$. The review of applications of the stokeslet method is in the work of [8]. The situation in the investigation of this problem is similar to one in quantum mechanics, where the singular solutions (zero-range potentials), which have been put into operation by E. Fermi $[9,10]$, became an instrument for the research of many complicated atomic systems. Thirty years ago F.A. Berezin and L.D. Faddeev [11] showed that from the mathematical point of view, the specification of the zero-range potential defines a selfadjoint extension of a symmetric operator. The basic advantage of the indicated method is the fact that it allows one to construct explicitly solvable models of complex objects. This operator approach gives one the application of the zero-range potential method for solving of many problems in absolutely different branches (from the theory of $N$-particles quantum systems to the theory of elasticity and diffraction theory [12 - 20]. Thus, the field of applications of zero-range potential method is expanded. It is possible because the operator approach reveals general (mathematical) features of these physical problems. That is why it is interesting to analyze the stokeslet from the point of view of the operator theory. 
A plane flow in two channels connected through a small opening is considered as an example of application of the suggested approach. A stokeslet is a model if a small obstacle for a flow. But the operator approach allows us to understand that a model of a small opening is essentially the same as a model of a small obstacle. Thus we have now an opportunity to study a flow in domains connected through a small opening. An example of such flow (two-dimensional flow between straight lines) is considered in the paper. The picture of streamlines in such two connected channels is obtained. It occurs that there is an infinite sequence of eddies. The existence of eddies far from an obstacle in creeping flow is known for many particular problems. Moffatt H.K. [21] examined two-dimensional Stokes flow in a corner formed by two intersecting rigid planes. In the same paper, Moffatt briefly considered two-dimensional Stokes flow between parallel planes which may be regarded as the limiting case of flow in a corner formed by two intersecting planes as the angle of the corner approaches zero. Moffatt showed that the dimensionless stream function $\Psi$ for the flow between parallel planes at $x= \pm 1$ induced by an unspecified two-dimensional disturbance centered at $y=0$ is

$$
\begin{aligned}
\Psi= & R e \sum_{n=1}^{\infty}\left[A_{n}\left(x \sin \left(\frac{1}{2} \lambda_{n} x\right)-\tan \left(\frac{1}{2} \lambda_{n}\right) \cos \left(\frac{1}{2} \lambda_{n} x\right)\right) e^{-\frac{1}{2 \lambda_{n}|y|}+}\right. \\
& \left.B_{n}\left(x \cos \left(\frac{1}{2} \mu_{n} x\right)-\cot \left(\frac{1}{2} \mu_{n}\right) \sin \left(\frac{1}{2} \mu_{n} x\right)\right) e^{-\frac{1}{2} \mu_{n}|y|}\right]
\end{aligned}
$$

for $y \neq 0$, where $A_{n}$ and $B_{n}$ are constants determined by the disturbance driving the flow, and $\lambda_{n}$ and $\mu_{n}$ satisfy the equations:

$$
\begin{gathered}
\sin \lambda_{n}+\lambda_{n}=0, \sin \mu_{n}-\mu_{n}=0, \\
\lambda_{n} \simeq 2^{-1}(4 n-1) \pi+i \log ((4 n-1) \pi), \\
\mu_{n} \simeq 2^{-1}(4 n+1) \pi+i \log ((4 n+1) \pi)
\end{gathered}
$$


The expansion for $\Psi$ is only implicit in [21], only the leading term is actually considered. Moffatt was the first to discover that the leading term in the expansion for $\Psi$ is associated with an infinite sequence of eddies on each side of the disturbance causing the flow. Later Hackborn [3] gave a more detailed description of such flow. It is shown in my paper that the analogous sequence of eddies is in the flow in two connected channels. The eddies farthest from the opening in figure 1 are essentially "Moffatt eddies" (or, more precisely, "Hackborn eddies"). The picture of flow in a case of periodic system of point-like openings is considered. It is interesting that there is an opportunity to change the character of the flow by varying the distance between the openings.

\section{STOKESLET}

Let us consider first of all a 2-dimensional problem. In this case it is convenient to introduce a stream function $\Psi$ satisfying the relations

$$
v_{1}=\frac{\partial \psi}{\partial x_{2}}, \quad v_{2}=-\frac{\partial \psi}{\partial x_{1}}
$$

Then the Stokes equations lead to the following equation for the function $\psi: \Delta^{2} \psi=0$. Let us define a stokeslet as a zero-range interaction for the biharmonic equation (the corresponding information concerning to the biharmonic equation is in [22]).

Let us consider the operator $\Delta_{0}^{2}$ in the space $L_{2}\left(\mathbb{R}^{2}\right)$ acted as the operator $\Delta^{2}$ and defined on the set of smooth finite functions from $L_{2}\left(\mathbb{R}^{2}\right)$ vanishing near the point zero. The closure of this operator is a symmetric operator with the domain

$$
\begin{array}{r}
D\left(\Delta_{0}^{2}\right)=\left\{u: u \in L_{2}\left(\mathbb{R}^{2}\right), \Delta^{2} u \in \mathrm{L}\left(\mathbb{R}^{2}\right),\right. \\
\left.u(0)=\frac{\partial u}{\partial x_{i}}(0)=\frac{\partial^{2} u}{\partial x_{i} \partial x_{j}}(0)=0, i, j=1,2\right\} .
\end{array}
$$

One can note that functions from $D\left(\Delta_{0}^{2}\right)$ are continuous, and its derivatives (of first and second order) are continuous too $\left(u \in C_{2, \text { loc }}^{2}\right)$ in accordance with imbedding theorems, that is the boundary conditions at the point $x=0$ are correct. 
One can see that the operator $\Delta_{0}^{2}$ has deficiency indices $(6,6)$. Indeed, let us consider the fundamental solution $g$ of the equation

$$
\Delta^{2} g(x)+k^{2} g(x)=\delta(x)
$$

corresponding to the regular point $k^{2}(k>0)$ of the operator $\Delta^{2}$,

$$
\begin{aligned}
g(x)= & \frac{1}{2 \pi} \int_{\mathbf{R}^{2}} \frac{\exp (i(\xi, x))}{\mid \xi^{4}+k^{2}} d \xi= \\
& =\frac{1}{8 k}\left(H_{0}^{(1)}(\sqrt{i k} r)+H_{0}^{(2)}(\sqrt{-i k} r)\right)
\end{aligned}
$$

and let us choose such derivatives $g_{x_{1} x_{2}}^{\left(j_{1}, j_{2}\right)}$, which belongs to the space $L_{2}\left(\mathbb{R}^{2}\right)$. This fact takes place if $j_{1}+j_{2} \leq 2$ (in this case the singularity at the point $x=0$ is sufficiently weak). It means that the deficiency indices are $(6,6)$.

To construct a self-adjoint extension it is necessary to describe the domain of the adjoint operator. Taking into account the asymptotics of the fundamental solution $g$ one can obtain that any element $u$ from the domain of the operator $\Delta_{0}^{2 *}$ takes a form:

$$
\begin{aligned}
u(x)= & \sum_{i, j=1}^{2} c_{i, j}^{u} g_{x_{i}, x_{j}}(x)+\sum_{i=1}^{2} c_{i}^{u} g_{x_{i}}(x)+c_{0} g(x)+ \\
& +\xi(x)\left(a_{0}-\sum_{i=1}^{2} a_{i} x_{i}+\sum_{i, j=1}^{2} a_{i j} g_{i j} x_{i} x_{j}\right)+u_{0}(x) .
\end{aligned}
$$

Here $u_{0} \in D\left(\Delta_{0}\right), g_{i j}=1, i \neq j, g_{i i}=2^{-1}, i, j=1,2, \xi(x)$ is a smooth cutting function: $\xi(x)=1,|x| \leq 1, \xi(x)=0,|x| \geq 2$. The function $\left(\Delta_{0}^{2 *} u\right)(x)$ at the point $x(x \neq 0)$ is computed as

$$
\left(\Delta_{0}^{2 *} u\right)(x)=\delta^{2} u(x) .
$$

The bilinear form 


$$
I(u, v)=\left(\Delta_{0}^{2 *} u, v\right)-\left(u, \Delta_{0}^{2 *} v\right)
$$

on the elements from $D\left(\Delta_{0}^{2 *}\right)$ can be computed by taking into account the representation (5):

$$
\begin{aligned}
I(u, v)= & a_{0}^{u} \overline{c_{0}^{v}}-c_{0}^{u} \overline{a_{0}^{v}}+\sum_{i=1,2}\left(a_{i}^{u} \overline{c_{i}^{v}}-c_{i}^{u}-\overline{a_{i}^{v}}\right)+ \\
& +\sum_{i, j=1,2}\left(a_{i j}^{u} \overline{c_{i j}^{v}}-c_{i j}^{u} \overline{a_{i j}^{v}}\right)
\end{aligned}
$$

It is necessary to select such linear subset of $D\left(\Delta_{0}^{2 *}\right)$, that the form $I$ annihilates on the elements of this subset to construct a domain of self-adjoint extension of the operator $\Delta_{0}^{2}$. It is an ordinary problem of linear algebra in a space $\mathbb{C}^{6}$. As a result we obtain

Theorem 1. The operator $\Delta_{s}^{2}$ (extension) is self-adjoint if and only if $\Delta_{0}^{2} \subset \Delta_{s}^{2} \subset \Delta_{0}^{2 *}$. Here $D\left(\Delta_{s}^{2}\right)$ is such linear subset of $D\left(\Delta_{0}^{2 *}\right)$ having no extensions that one of the following conditions is valid for the boundary vectors of any function from the set $D\left(\Delta_{s}^{2}\right)$

1) $U=\left(U_{0}, U_{1}\right), U_{0}=A U_{1}, U_{1}=\left(c_{0}^{u}, c_{1}^{u}, c_{2}^{u}, c_{11}^{u}, c_{12}^{u}, c_{22}^{u}\right)$, $U_{0}=\left(a_{0}^{u}, a_{1}^{u}, a_{2}^{u}, a_{11}^{u}, a_{12}^{u}, a_{22}^{u}\right), A: \mathbb{C}^{6} \rightarrow \mathbb{C}^{6}, A=A^{*}$,

2) $U_{1}=A U_{0}, A: \mathbb{C}^{6} \rightarrow \mathbb{C}^{6}, \mathrm{~A}=\mathrm{A}^{*}$,

3) $U_{0}=\alpha+\gamma, U_{1}=\beta+A \gamma$, here $\alpha, \beta$ - are vectors from an arbitrary orthogonal fixed subspaces $N^{+}$and $N^{-}, N^{+}, N^{-} \in \mathbb{C}^{6}, \gamma \in N, N=$ $\mathbb{C}^{6} \ominus \mathrm{N}^{+} \ominus \mathrm{N}^{-}, A: N \rightarrow N$, the operator $A$ is self-adjoint and reversible.

It is more convenient very often to describe an extension using the coefficients of the asymptotics of functions near the point zero and not the coefficients of the expression (5). Using a well-known asymptotics of the fundamental solution $g$ one can show that an element $u$ from the set $D\left(\Delta_{0}^{2 *}\right)$ has the following asymptotics near the point zero: 


$$
\begin{aligned}
\left.u\right|_{|x| \rightarrow 0}= & c_{0} r^{2} \ln r+2 c_{1} r(\ln r) \cos \theta+ \\
& +2 c_{2} r(\ln r) \sin \theta+c_{11}\left(2 \ln r+1+2 \cos ^{2} \theta\right)+ \\
& +c_{12} \sin 2 \theta+c_{22}\left(2 \ln r+1+2 \sin ^{2} \theta\right)+\tilde{a}_{0}- \\
& -\tilde{a}_{1} r \cos \theta-\tilde{a}_{2} r \sin \theta+2^{-1} \tilde{a}_{11} r^{2} \cos ^{2} \theta+ \\
& +2^{-1} \tilde{a}_{12} r^{2} \sin 2 \theta+2^{-1} \tilde{a}_{22} r^{2} \sin ^{2} \theta+o\left(r^{2}\right)
\end{aligned}
$$

here $r \cos \theta=x_{1}, r \sin \theta=x_{2}$, the coefficients $c_{0}, c_{i}, c_{i j}$ coincides with the corresponding coefficients from (5), the vector $\tilde{U}_{0}, \bar{U}_{0}=\left\langle\tilde{a}_{0}, \tilde{a}_{1}, \tilde{a}_{2}\right.$, $\left.\tilde{a}_{11}, \tilde{a}_{12}, \tilde{a}_{22}\right)$, satisfies the following correlation: $\tilde{U}_{0}=U_{0}+B U_{1}$, here $B$ is a self-adjoint operator in $\mathbb{C}^{6}$, which is determined by the asymptotics of function $g$ near the point zero. Using this notation we can reformulate our statement:

Theorem 1'. The operator $\Delta_{s}^{2}$ is self-adjoint if and only if $\Delta_{0}^{2} \subset$ $\Delta_{s}^{2} \subset \Delta_{0}^{2 *}$, here $D\left(\Delta_{s}^{2}\right)$ is such linear subset of $D\left(\Delta_{0}^{2 *}\right)$, having no extensions, that one of the following conditions is valid for the boundary vector $\tilde{U}$ of any function from the set $D\left(\Delta_{s}^{2}\right)$

1) $\tilde{U}=\left(\tilde{U}_{0}, U_{1}\right), \tilde{U}_{0}=A U_{1}, A: \mathbb{C}^{6} \rightarrow \mathbb{C}^{6}, \mathrm{~A}=\mathrm{A}^{*}$,

2) $U_{1}=A \tilde{U}_{0}, A: \mathbb{C}^{6} \rightarrow \mathbb{C}^{6}, \mathbf{A}=\mathbf{A}^{*}$,

3) $\tilde{U}_{0}=\alpha+\gamma, U_{1}=\beta+A \gamma$, here $\alpha, \beta$ are such as has been described above.

Various hydrodynamics applications of stokeslets are described in the review of Hasimoto H., Sano O. [8]. Let us discuss a relation between the solution of the model problem constructed in the framework of self-adjoint operator extension theory and the solution of corresponding "realistic" problem. We shall make a comparison using an example of such problem- a problem of description of Stokes flow past a cylinder (a 
curve in $\mathbb{R}^{2}$ ). This ("realistic", not "model") problem was considered in [2]. The flow is determined by the following correlation far from a body:

$$
\mu\left(w-w_{0}\right)=\mu w_{0}+\lambda \bar{c}+\chi c+O\left(x^{-1}\right),
$$

here

$$
\begin{aligned}
\mu w_{s} & =\bar{c} \ln (z \bar{z})-c \bar{z} z^{-1}, \\
z & =x_{1}+i x_{2}, w==v_{1}-i v_{2},
\end{aligned}
$$

$w=w_{0}$ is a boundary condition on the cylinder, constant values $\lambda$ and $\chi$ are determined by a shape and a size of the cylinder. The value $c$ is computed by the matching of this solution with an external flow [23] having the following internal asymptotics:

$$
\mu w=\mu w_{s}+\lambda_{\infty} \bar{c}+\chi_{\infty} c .
$$

Hence

$$
c=\frac{\mu\left[\left(\lambda_{\infty}-\lambda\right) \bar{w}_{0}-\left(\bar{\chi}_{\infty}-\bar{\chi}\right) w\right]}{\left|\lambda_{\infty}-\lambda\right|^{2}-\left|\chi_{\infty}-\chi\right|^{2}} .
$$

The parameters $\lambda$ and $\chi$ have been computed for some bodies [2]. For example, $\lambda=-2 \ln a, \chi=\sigma^{2}$ for an elliptic cylinder with halfaxes $a\left(1+\sigma^{2}\right)$ and $a\left(1-\sigma^{2}\right)$. The value $\lambda$ is called usually the parameter of equivalent circular cylinder and $\chi$ is called the parameter of the equivalent elliptic cylinder.

One can consider a model problem to describe a flow past a stokeslet. It should be mentioned that this problem has a solution in an explicit form (as has been described above) and the form is the same as (7). It is easy to show that there exist such values of the extension parameters that the model solution coincides with the asymptotics (far from a body) of the solution of the "realistic" problem. The problem of the parameters $\lambda$ and $\chi$ determination is in such a way the problem of choosing of the extension parameters from the point of view of the operator theory. 


\section{DISTOKESLET}

The described operator scheme does not include "higher stokeslets" because the corresponding solutions have a strong singularity at the point zero and don't belong to the space $L_{2}$ But one can realize the operator approach in this situation too. It is necessary to expand the initial space by adding the corresponding singular solutions. Unfortunately, this way leads to an indefinite metric space, but this fact does not destroy the construction. Let us describe how to take into account a distokeslet. We shall use an approach analogous to one in $[24,20]$.

Let $A_{1}$ be the following set of functions:

$$
\begin{aligned}
A_{1}= & \left\{f(x): f \in L_{2}\left(\mathbb{R}^{2}\right), \Delta^{2} f \in \mathrm{L}_{2}\left(\mathbb{R}^{2}\right),\right. \\
& \left.\int_{\mathbf{R}^{2}} f(x)\left|x-x_{0}\right|^{-4} d x-\text { converges }\right\} . \\
h_{-1}(x)= & g^{\left(j_{1}, j_{2}\right\rangle}(x), h_{1}=\left(\Delta^{2}-\lambda_{0}\right)^{-1} h_{-1},
\end{aligned}
$$

where $\lambda_{0}$ is some negative value (a regular point of the operator $\Delta^{2}$ ), $g^{\left(j_{1}, j_{2}\right)}(x)$ is the corresponding derivative of the fundamental solution $g$. Let $\mathcal{U}_{1}$ be a set of elements which can be represented in a form

$$
f=f_{1}+c_{1} h_{1}+c_{-1} h_{-1},
$$

where $f \in A_{1}$. We define a scalar product in $\mathcal{U}_{1}$ by the following expression:

$$
\begin{aligned}
(f, \varphi) u_{1}=\left(f_{1}, \varphi_{1}\right)_{A_{1}} & +c_{1}^{f} \int_{\mathbf{R}^{2}} h_{1} \overline{\varphi_{1}} d x+\overline{c_{1}^{\varphi}} \int_{\mathbf{R}^{2}} f_{1} \overline{h_{1}} d x+ \\
& +\left(c_{1}^{f} \overline{c_{-1}^{\varphi}}+c_{-1}^{f} \overline{c_{1}^{\varphi}}\right) \int_{\mathbf{R}^{2}} h_{1} \overline{h_{-1}} d x
\end{aligned}
$$


The topology in the set $\mathcal{U}_{1}$ is defined by a standard manner [25]. The form $(f, \varphi) \mathcal{U}_{1}$ should be transformed to a diagonal form. The obtained expression contains one "negative square". One obtains a positive form by replacing the corresponding sign "minus" by "plus". This form gives an expression for a positively definite scalar product, which is closely linked with a topology. The closure of the set $\mathcal{U}_{1}$ in a space with such topology is a Pontryagin's space $\Pi_{1}$.

Let us consider the operator $\bar{\Delta}^{2}$ with the domain

$$
D\left(\tilde{\Delta}^{2}\right)=\left\{f \in \tilde{A}_{1}, f_{1} \in W_{2}^{4, \text { loc }}, f_{1}=f_{2}+c h_{1}, h_{-1} \notin D\left(\tilde{\Delta}^{2}\right)\right\}
$$

where $f_{2}$ is such function from $A_{1}$, that

$$
\left(\tilde{\Delta}^{2}-\lambda_{0}\right) f_{2} \in \mathcal{U}_{1} \text {. }
$$

The operator $\tilde{\Delta}^{2}$ acts as a square of the Laplace operator on the set $A_{1}$, and the image of the element $h_{1}$ is such that

$$
\left(\tilde{\Delta}^{2}-\lambda_{0}\right) h_{1}=h_{-1} \text {. }
$$

The operator $\tilde{\Delta}^{2}$ is a symmetric one. More over it is a self-adjoint one because the following relation takes place:

$$
\left(\tilde{\Delta}^{2}-\lambda_{0}\right) D\left(\Delta^{2}\right)=\mathcal{U}_{1}
$$

Remark. One can note that not only $\lambda_{0}$, but also the whole of a negative half-axis belongs to the set of regular points of the operator $\tilde{\Delta}^{2}$. The construction only depends on the choice of the parameter $\lambda_{0}$, but the space $\mathcal{U}_{\mathbb{1}}$ does not depend on it.

Let $\Delta_{1,0}^{2}$ be a restriction of the operator $\bar{\Delta}^{2}$ onto the set

$$
D\left(\Delta_{1,0}^{2}\right)=\left\{f: f \in D\left(\Delta_{0}^{2}\right), \quad\left(\left(\tilde{\Delta}^{2}-\lambda_{0}\right) f, h_{-1}\right)=0\right\} .
$$

The obtained operator $\Delta_{1,0}^{2}$ is a symmetric one and has deficiency indices $(1,1)$. Indeed, the condition (8) means that the element $h_{-1}$ is 
orthogonal to the set of images of the operator $\bar{\Delta}^{2}-\lambda_{0}$. There are no other deficiency elements because the set $\mathcal{U}_{1}$ is a dense subset of the space $\mathrm{II}_{1}$ in respect to the described topology.

It is necessary to study a "boundary form" $J$ for the adjoint operator

$$
J(f, \varphi)=\left(\left(\Delta_{1,0}^{2 *}-\lambda_{0}\right) f, \varphi\right)-\left(f,\left(\Delta_{1,0}^{2 *}-\lambda_{0}\right) \varphi\right), f, \varphi \in D\left(\Delta_{1,0}^{2 *}\right)
$$

to construct a self-adjoint extension. Let us obtain the following expression for the form $J$ :

$$
J(f, \varphi)=c_{1}^{f} \overline{c_{-1}^{\varphi}}-c_{-1}^{f} \overline{c_{1}^{\varphi}}
$$

here

$$
c_{1}^{f}=\left(\left(\bar{\Delta}^{2}-\lambda_{0}\right) f_{1}, h_{-1}\right)
$$

Indeed, an element $f$ from the set $D\left(\Delta_{1,0}^{2 *}\right)$ can be represented in a form $f=\tilde{f}+c_{-1} h_{-1}$, here $\tilde{f} \in D\left(\bar{\Delta}^{2}\right)$. The definition of a scalar product in $\mathcal{U}_{1}$ causes the following relationship

$$
\left(\left(\bar{\Delta}^{2}-\lambda_{0}\right) h_{1}, h_{-1}\right)=0 .
$$

The operator $\Delta_{1,0}^{2 *}$ acts as $\tilde{\Delta}^{2}$ on the set $D\left(\tilde{\Delta}^{2}\right)$. We obtain now the expression for the boundary form $J$ by taking into account a self-adjointness of the operator $\tilde{\Delta}^{2}$.

Remark. The coefficient $c_{1}^{f}$ is related with the asymptotics of function $f$ near the point $x_{0}$. One can get this relationship by a transformation of the expression (9) in accordance with the Green's formula:

$$
\begin{aligned}
c_{1}^{f}= & \left(\left(-\bar{\Delta}^{2}-\lambda_{0}\right) f_{1}, h_{-1}\right)=\int_{\Sigma_{\varepsilon}}\left(\frac{\partial h_{-1}}{\partial n} \Delta f-\frac{\partial f}{\partial n} \Delta h_{-1}+\right. \\
& +\frac{\partial \Delta h_{-1}}{\partial n} f-\frac{\partial \Delta f}{\partial n} h_{-1}-\frac{\partial f}{\partial x_{1}} \frac{\partial}{\partial x_{1}}\left(\frac{\partial h_{-1}}{\partial n}\right)+
\end{aligned}
$$




$$
\begin{aligned}
& +\frac{\partial h_{-1}}{\partial x_{1}} \frac{\partial}{\partial x_{1}}\left(\frac{\partial f}{\partial n}\right)+f \frac{\partial^{2}}{\partial x_{2}^{2}}\left(\frac{\partial h_{-1}}{\partial n}\right)- \\
& \left.-h_{-1} \frac{\partial^{2}}{\partial x_{2}^{2}}\left(\frac{\partial f}{\partial n}\right)\right) d s, \Sigma_{\varepsilon}=\left\{x:\left|x_{0}-x\right|=\varepsilon\right\} .
\end{aligned}
$$

It is necessary to find a linear subset of the set $D\left(\Delta_{1,0}^{2 *}\right)$ such that the form $J$ annihilate on this subset to construct a domain of the operator $\Delta_{1,0}^{2}$. It is a simple problem of linear algebra similar to one in the second paragraph. This investigation results in the following description of the domain in question:

Theorem 2. The domain of self-adjoint extension of the operator $\Delta_{1,0}^{2}$ consists of all elements from the set $D\left(\Delta_{1.0}^{2 *}\right)$, satisfying the condition $c_{1}=a c_{-1}$, where $a$ is a real number.

This result is the description of distokeslet or more precisely of one element of the distokeslet family (with fixed orientation). One can use this operator approach for the description of other distokeslets and also for stokeslets of higher orders.

Let us remark, how to change this scheme if we deal with a 3dimensional problem. In this case the Stokes equations can be represented in the following matrix form:

$$
A\left(\begin{array}{c}
p \\
\vec{v}
\end{array}\right)=\left(\begin{array}{l}
0 \\
\overrightarrow{0}
\end{array}\right)
$$

here

$$
A=\left(\begin{array}{cc}
-\vec{\nabla} & \mu \Delta \\
0 & -\vec{\nabla}
\end{array}\right), \quad \vec{v}=\left(\begin{array}{c}
v_{1} \\
v_{2} \\
v_{3}
\end{array}\right) .
$$

Let us consider the operator $A^{2}$,

$$
A^{2}=\left(\begin{array}{cc}
\Delta & \mu \Delta \vec{\nabla}+\Delta \\
0 & \Delta
\end{array}\right)
$$

on the set of elements satisfying the condition $\nabla \cdot \vec{v}=0$. On this set the equation reduces to the system of four Laplace equations (for every component). That is we can construct the model for the diagonal operator 
$\operatorname{diag}\{\Delta, \Delta, \Delta, \Delta\}$ in a space of vector-functions $\left(p, v_{1}, v_{2}, v_{3}\right)$. Hence, the construction of the model reduces to the standard zero-range potential model (for the vector-functions). This model can be constructed not only in the space $L_{2}$ (see, for example, $[13,14]$ ), but also in an extended space [20]. It should be noted that the model is constructed here for the operator $A^{2}$ and not for the operator $A$, that is why it is necessary to examine if our singular model function is a solution of the initial equations or not. It is easy to show that the singular solution (2) (see Introduction) is a model solution in the case when a dipole is taken into account in the model Laplace operator.

\section{CREEPING FLOW IN TWO CONNECTING CHANNELS}

In this section we shall describe one example of application of suggested approach. Let us consider two-dimensional flow in a channel $\Omega^{+}$ with straight boundaries $(x=2, x=0)$ which is connected with the identical channel $\Omega^{-}(x=-2, x=0)$ through the opening at the point $(0,0)$. Here $x, y$ are Cartesian coordinates of a point. We suppose that the following boundary condition is valid: $\psi=\frac{\partial \psi}{\partial n}=0, x= \pm 2,0$. The described operator version of stokeslet approach can be modified for the case when a singularity is at the boundary. Let us describe the modification of the model for this situation. We shall consider the square of the Laplace operator with the boundary condition mentioned above. Let $\left(\Delta_{0}^{+}\right)^{2}$ be the restriction of the initial operator onto the set of smooth functions, which satisfy the following condition near the point $(0,0)=r_{0}$ of the boundary:

$$
\begin{aligned}
D\left(\Delta_{0}^{+^{2}}\right)= & \left\{u: u \in L_{2}\left(\Omega^{+}\right), \Delta^{2} u \in L\left(\Omega^{+}\right),\right. \\
& \left.u\left(r_{0}\right)=u_{x_{i}}^{\prime}\left(r_{0}\right)=u_{x_{j} x_{i}}^{\prime \prime}\left(r_{0}\right)=0\right\} .
\end{aligned}
$$

The deficiency elements may be obtained by the following procedure. Let $r_{0}^{\prime}$ be an internal point of $\Omega^{+}$. One can find the solution of the problem:

$$
\Delta^{2} g(r)+k^{2} g(r)=\delta\left(r-r_{0}^{\prime}\right),\left.g(r)\right|_{\partial \Omega}=\left.g_{n}^{\prime}\right|_{\partial \Omega}=0 .
$$

Here $k^{2}>0$. The asymptotics of the solution near the point $r_{0}^{\prime}$ is the same as one of the fundamental solution. It is necessary to look for the solution $g$ and its derivatives when $r_{0}^{\prime} \rightarrow r_{0}$. It occurs that the function 
$\frac{\partial^{2} g}{\partial n_{r^{\prime}}^{2}}$ only gives a non-zero limit (non-zero derivatives of higher orders don't belong to the space $L_{2}$ ). This function is the deficiency element of the operator $\left(\Delta_{0}^{+}\right)^{2}$. That is, the deficiency indices of the operator $\left(\Delta_{0}^{+}\right)^{2}$ are $(1,1)$. One can show that the main term of the asymptotics of the deficiency element near the point $r_{0}$ is $c \cos ^{2} \theta$, where $\theta$ is the angle between the vector $r-r_{0}$ and the normal at the point $r_{0}$.

The analogous construction (the operator $\left(\Delta_{0}^{-}\right)^{2}$ ) is for the second channel. Let the operator $\Delta_{0}^{2}$ be the orthogonal sum of these operators. It is the symmetric one with the deficiency indices $(2,2)$. The domain of the adjoint operator consists of the following elements:

$$
u=\left(u^{ \pm}, u^{ \pm}\right), u^{ \pm}=\alpha^{ \pm} w^{ \pm}+\beta^{ \pm} v^{ \pm}+u_{0}^{ \pm}
$$

where

$$
w^{ \pm}=2^{-1}\left(h_{\lambda}^{ \pm}+h_{\lambda}^{ \pm}\right), v^{ \pm}=2^{-1}\left(h_{\lambda}^{ \pm}-h_{\bar{\lambda}}^{ \pm}\right),
$$

$\lambda=k^{2}$ is a complex regular point for the operators $\Delta_{0}^{ \pm}, h_{\lambda}^{ \pm}$is the corresponding deficiency element, $u_{0}^{ \pm} \in D\left(\Delta_{0}^{ \pm}\right)$. One can obtain the boundary form $I$ for the adjoint operator:

$$
I(f, \phi)=\alpha_{f}^{+} \overline{\beta_{\phi}^{+}}-\beta_{f}^{+} \overline{\alpha_{\phi}^{+}}+\alpha_{f}^{-} \overline{\beta_{\phi}^{-}}-\beta_{f}^{-} \overline{\alpha_{\phi}^{-}}
$$

The domain of self-adjoint extension is a linear subset of the domain of the adjoint operator, on the elements of which the boundary form annihilates. It is easy to prove

Theorem 3. The domain of self-adjoint extension consists of all elements from the domain of the adjoint operator, which satisfy the condition:

$$
\left(\begin{array}{l}
\beta^{-} \\
\beta^{+}
\end{array}\right)=A\left(\begin{array}{c}
\alpha^{-}(s) \\
\alpha^{+}(s)
\end{array}\right) \text { or }\left(\begin{array}{c}
\alpha^{-}(s) \\
-\beta^{+}(s)
\end{array}\right)=B\left(\begin{array}{c}
\beta^{-}(s) \\
\alpha^{+}(s)
\end{array}\right)
$$

Here $A$ and $B$ ane Hermitian matrices.

Remark. The richness of the extensions' family (e.g., the fact that the number of the deficiency elements for the case of singularity inside 
the domain is sufficiently large, see Section 2) allows us to simulate different physical situation. If we choose, for instance, the extension for which the main singularity of the element from it's domain is a $\ln r$, we obtain "a source of eddy" (a more common name for this singularity is "line vortex" or "line rotlet"), and one can use it to describe, for example, a flow due to a small rotating body. A conventional stokeslet is represented by the singularity $r \ln r \cos \theta$. In the case of the singularity of the boundary we have the following main term of the asymptotics: $c_{ \pm} \cos ^{2} \theta$, we obtain (when $c_{+} c_{-}<0$ ) "a source of mass" for one channel and "a point sink" for the second one, hence, one can use this type of singularity to simulate the flow through a small aperture. In this section we deal with the extension of this type.

There is not necessity to describe thoroughly all extensions of the same type (and we shall not do it) to obtain a result (mentioned above) that there exist a series of eddies. Only the intensity of eddies depends on the choice of the extension, but its sizes and the geometry of the flow does not depend on it. Let us fix an extension. One can get the corresponding stream function (singular solution of the biharmonic equation) using a Fourier transform:

$$
\psi=2 \pi R e \sum_{n=1}^{\infty}\left(A_{n}(x \pm 1) e^{-\frac{1}{2} \lambda_{n}|v|}+B_{n}(x \pm 1) e^{\frac{-1}{2} \mu_{n}|y|}\right)
$$

Here the sign "-" corresponds to the first channel, the sign "+" corresponds to the second one.

$$
\begin{aligned}
& A_{n}(x)=\frac{x \sin \left(2^{-1} \lambda_{n} x\right) \cos \left(2^{-1} \lambda_{n}\right)-\sin \left(2^{-1} \lambda_{n}\right) \cos \left(2^{-1} \lambda_{n} x\right)}{\cos \left(\lambda_{n}\right)+1}, \\
& B_{n}(x)=\frac{x \cos \left(2^{-1} \mu_{n} x\right) \sin \left(2^{-1} \mu_{n}\right)-\cos \left(2^{-1} \mu_{n}\right) \sin \left(2^{-1} \mu_{n} x\right)}{\cos \left(\mu_{n}\right)-1},
\end{aligned}
$$

$\lambda_{n}$ and $\mu_{n}$ are the roots of the equations:

$$
\sin \left(\lambda_{n}\right)+\lambda_{n}=0, \quad \sin \mu_{n}-\mu_{n}=0 .
$$


One can obtain now a picture of streamlines for the flow as a simple consequence of this consideration (see figure 1). We suppose that the unperturbed solution is following: a flow with a stream function

$$
\psi_{0}=\gamma\left(4^{-1} x^{2}-8^{-1} x^{3}\right), 0 \leq x \leq 2
$$

in the right channel caused by moving of the channel boundary $(x=2)$ with constant velocity (i.e. $\psi_{0}$ satisfies the boundary conditions $\psi_{0}=$ $\frac{\partial \psi_{0}}{\partial n}=0, x=0, \psi_{0}=0, \frac{\partial \psi_{0}}{\partial \eta}=$ const., $x=2$ ) and the absence of flow in the left one. While computing streamlines we suppose that $\gamma=1$, $\lambda_{n}, \mu_{n}$ for $n>5$ are given by the asymptotic formulae (4) and first five numbers $\lambda_{n}, \mu_{n}$ are computed approximately by solving the equations (3) (these values are, for example, in [26], see table 1). The approximate solutions of the equations (3) is obtained by the complex form of Newton's method. Namely, let $H(z)=\sin z+z$ and $z_{j}$ be an approximation of the root of the equation $H(z)=0$. The next approximation is

$$
z_{j+1}=z_{j}-H\left(z_{j}\right)\left(H^{\prime}\left(z_{j}\right)\right)^{-1} .
$$

\begin{tabular}{|c|c|c|}
\hline$\frac{n}{1}$ & $\frac{\lambda_{n}}{4.21239+2.25073 \mathrm{i}}$ & $\frac{\mu_{n}}{7.49768+2.76858 \mathrm{i}}$ \\
2 & $10.7125+3.10315 \mathrm{i}$ & $13.9000+3.35221 \mathrm{i}$ \\
3 & $17.0734+3.55109 \mathrm{i}$ & $20.2385+3.71677 \mathrm{i}$ \\
4 & $23.3984+3.85880 \mathrm{i}$ & $26.5545+3.98314 \mathrm{i}$ \\
5 & $29.7081+4.09370 \mathrm{i}$ & $32.8597+4.19325 \mathrm{i}$ \\
\hline
\end{tabular}

Table 1. First roots of the equations

$$
\sin \left(\lambda_{n}\right)+\lambda_{n}=0, \sin \mu_{n}-\mu_{n}=0 .
$$

Starting with the asymtotic form for $\lambda_{n}$, it was found that only two or three iterations were necessary to obtain eight-figure accuracy. The analogous consideration is for the second equation.

The streamlines of the flow depicted in figure 1 are computed using a simple superposition of the unperturbed flow $\psi_{0}$ and the stream function $\psi$ in equation (10). One can see that the far-field flow in the first channel is similar to the unperturbed flow (without opening), and 
the far-field flow in the second channel (where a flow is absent without opening) consists of an infinite set of eddies which are separated by zero streamlines. Each eddy of the far-field flow has a length (in the $y$ direction) of about $2 \pi\left(\operatorname{Im} \lambda_{1}\right)^{-1} \simeq 2.8$ : moreover, owing to the presence of the exponential factor in the expression (10), the flow at corresponding points in adjacent eddies (i.e. at points with the same $x$-coordinate but differing by approximately $2 \pi\left(\operatorname{Im} \lambda_{1}\right)^{-1}$ in the $y$-coordinate) differs in speed by a factor approximately of $\exp \left(\pi \operatorname{Re} \lambda_{1}\left(\operatorname{Im} \lambda_{1}\right)^{-1}\right) \cong 360$ and is in opposite directions.

Remark. Of course, one can consider another profile $\hat{\psi}_{0}$ of the unperturbed flow. Then due to the linearity of the problem the general solution will be $\hat{\psi}_{0}+\psi$.

Let us consider now the situation when there are periodic set of point-like apertures at the points $(0, n a), n$ is an integer. The solution of model problem can be constructed in an explicit form in this case too. The stream function on one period $(0<y<a)$ is

$$
\begin{aligned}
\psi= & 2 \pi R e \sum_{n=1}^{\infty}\left(A _ { n } ( x \pm 1 ) \left(\exp \left(-2^{-1} \lambda_{n} y\right)+\right.\right. \\
& \left.+2 \exp \left(-2^{-1} \lambda_{n} a\right)\left(1-\exp \left(-2^{-1} \lambda_{n} a\right)\right)^{-1} \operatorname{ch}\left(2^{-1} \lambda_{n} y\right)\right)+ \\
& +B_{n}(x \pm 1)\left(\exp \left(-2^{-1} \mu_{n} y\right)+\right. \\
& \left.\left.+2 \exp \left(-2^{-1} \mu_{n} a\right)\left(1-\exp \left(-2^{-1} \mu_{n} a\right)\right)^{-1} \operatorname{ch}\left(2^{-1} \mu_{n} y\right)\right)\right)
\end{aligned}
$$

The computation of streamlines is analogous to the previous one. The character of the flow depends on the ratio of the period $a$ to the asymptotic period of eddies (which is related with the width of the channel). For the big period $(a=10)$ the flow in the left channel consists of series of eddies (figure 2), which are separated by zero streamlines as for the case of one opening. But for smaller values of the period the separating zero streamlines are less numerous (figures 3 ), and further decreasing of the period causes the appearance of a flow without eddies near the wall 
opposite to the openings (figure 4, $a=2$ ). We do not draw the picture of streamlines in the right channel because there is a simple superposition of flow similar to one in the left channel and the main unperturbed flow.

Acknowledgements. The work is supported by the grant No 94-2.71067 of the Ministry of Higher Education of Russia, No 96-01-00074 and No 95-01-00439 of RFBR, Soros Foundation and ANS RF. The author thanks Prof. Yu.V.Gugel for the assistance in carrying out the calculations.

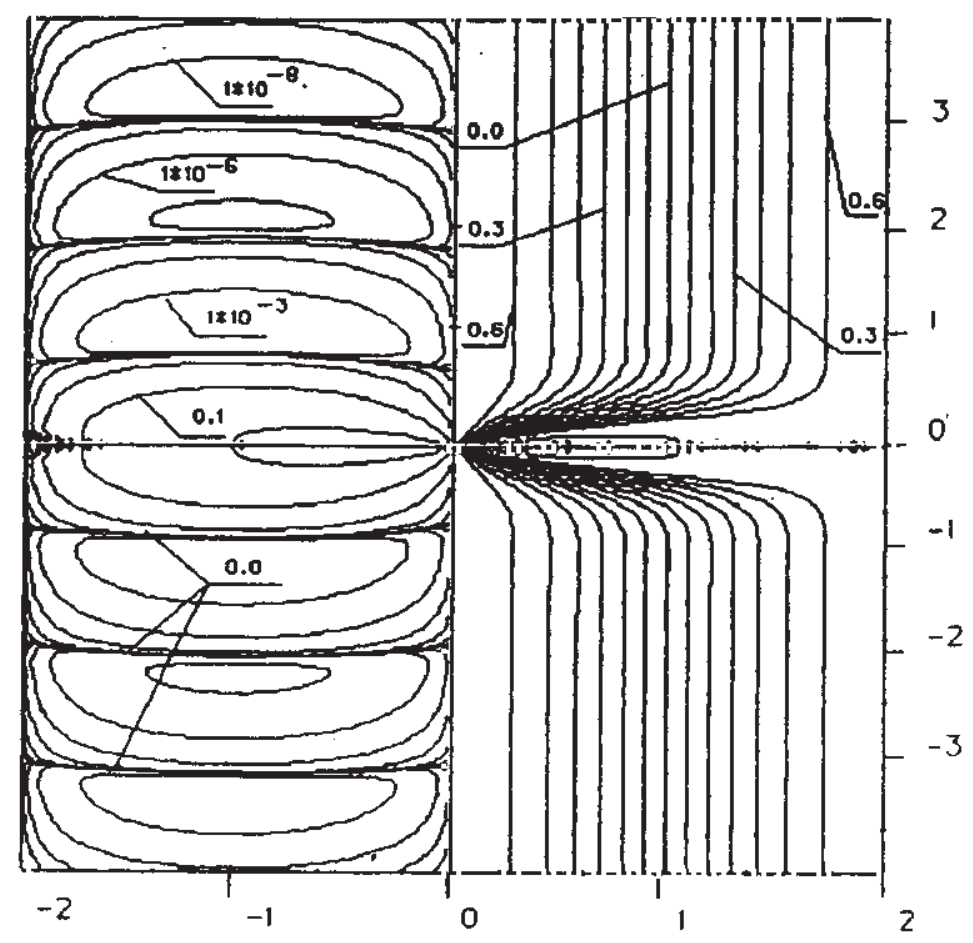

Fig. 1. The picture of the streamlines for the case of one aperture. The values of $\psi$ is indicated. 


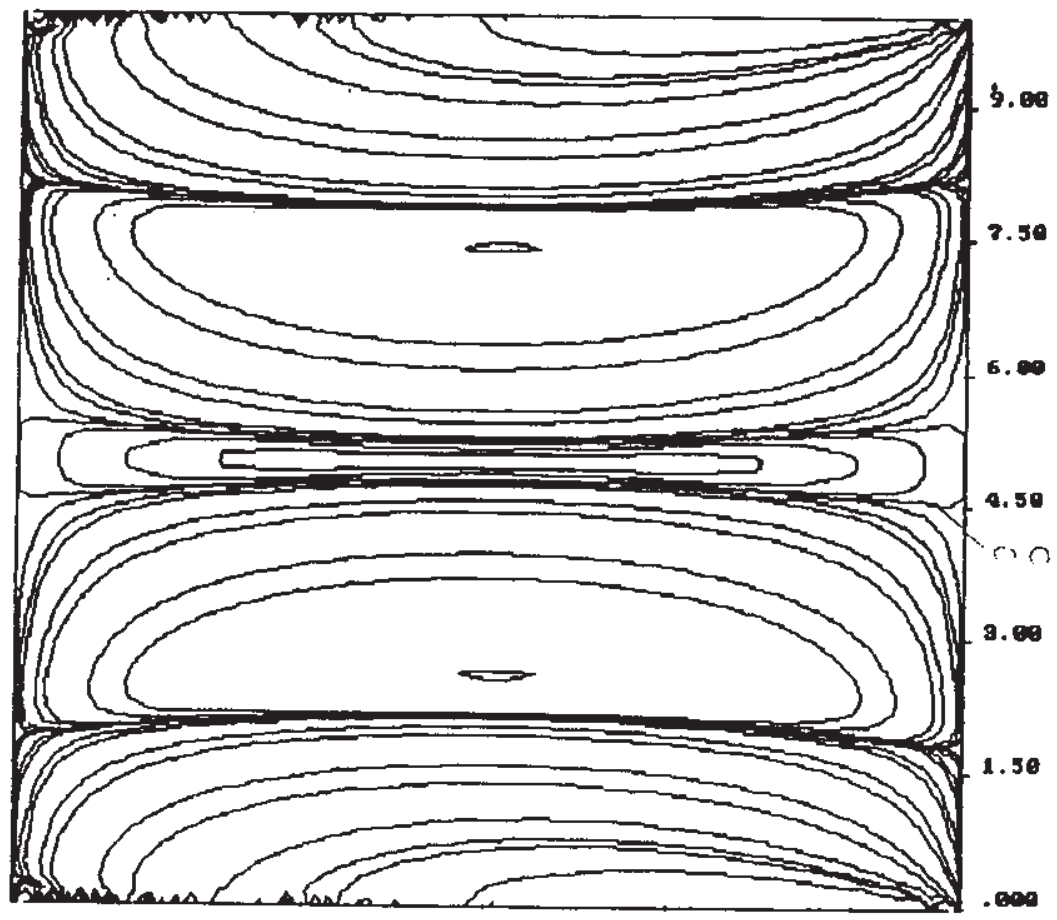

Fig. 2. The picture of the streamlines for the case of periodic system of apertures. $a=10$. 


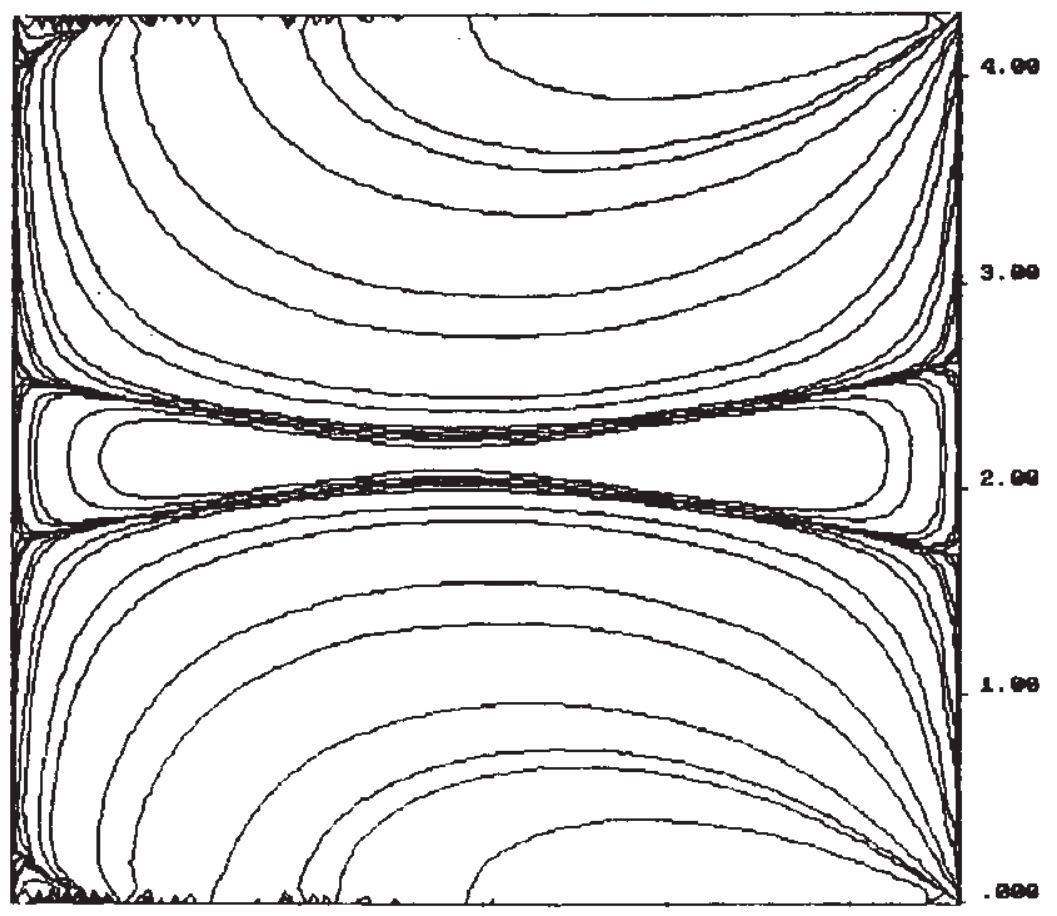

Fig. 3. The picture of the streamlines for the case of periodic system of apertures. $a=4.25$. 


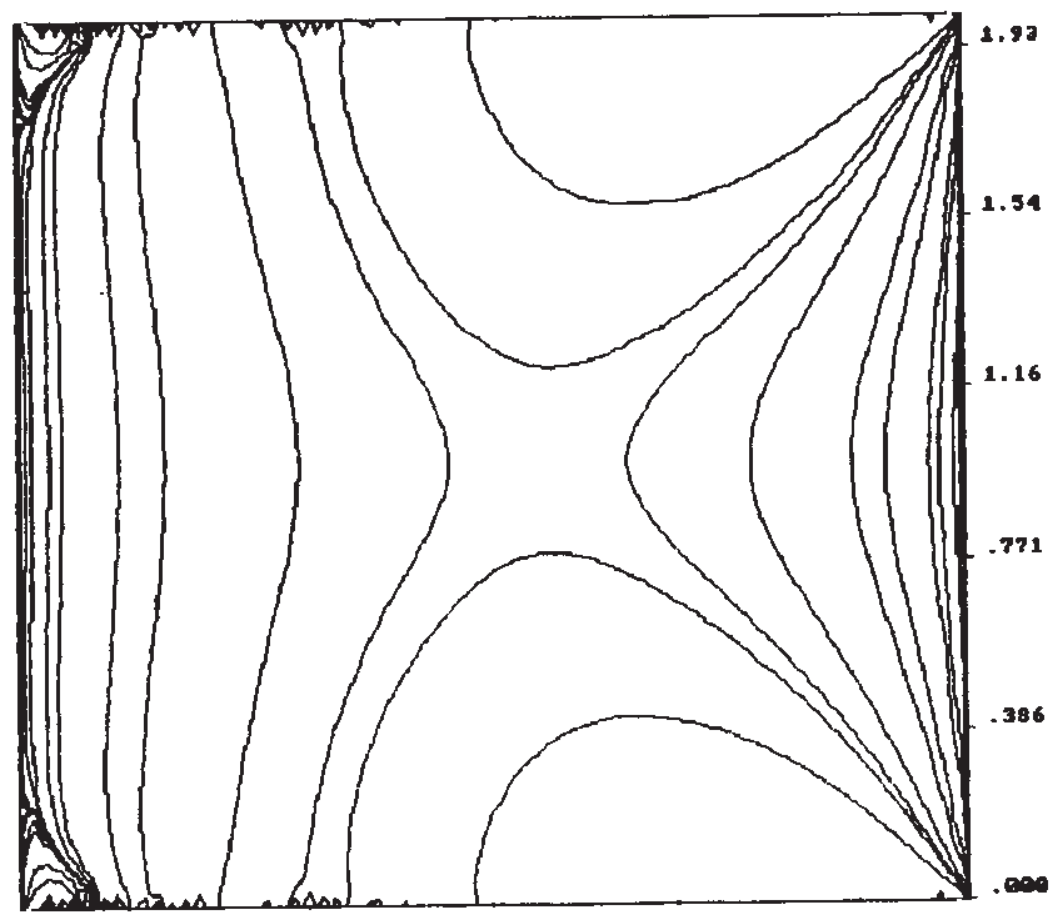

Fig. 4. The picture of the streamlines for the case of periodic system of apertures. $a=2$. 


\section{References}

[1] Hancock, G.J., The self-propulsion of microscopic organisms through liquids, Proc. R. Soc. London. Ser. A. 217 (1953), 96-121.

[2] Batchelor, G.K., Slender-body theory for particles of arbitrary crosssection in Stokes flow, J. Fluid Mech. 44 (1970), 419-440.

[3] Hackborn, W.W., Asymmetric Stokes flow between parallel planes due to a rotlet, J. Fluid Mech. 218 (1990), 531-546.

[4] Liron, N. and Shahar, R., Stokes flow for a Stokeslet between two parallel flat plates. J. Eng. Math. 10 (1976), 287-303.

[5] Liron, N. and Shahar, R., Stokes flow due to a stokeslet in a pipe, J. Fluid Mech. 86 (1978), 727-744.

[6] Blake, J.R. and Chwang, A.T., Fundamental singularities of viscots flow. Part 1. The image system in the vicinity of a stationary no-slip boundary, J. Eng. Math. 8 (1974), 23-29.

[7] Chwang, A.T. and Wu, T.Y., Hydromechanics of low-Reynolds-number flow. Part 2. Singularity method for Stokes flows, J. Fluid Mech. 67 (1975), 787-815.

[8] Hasimoto, H. and Sano, O., Stokeslets and eddies in creeping flow, Ann. Rev. Fluid Mech. 12 (1980), 335-363.

[9] Fermi, E., Sopra lo spostamento per pressione delle righe elevate delle serie spettrali, Nuovo Cim. 11 (1934), 157-166.

[10] Fermi, E., Sul moto dei neutroni nelle sostanze idrogenate, Ric. Sci. 7 (1936), 13-52. 
[11] Berezin, F.A. and Faddeev, L.D., Remarks on the Schrödinger equation with singular potentials, Doklady AN SSSR 137, No 5 (1961), 10111014 (in Russian); English transl. in Soviet Math. Dokl. 2 (1961).

[12] Albeverio, S., Fenstad, J.E., Høegh-Krohn, R. and Lindstrom, T., Nonstandard Methods in Stochastic Analysis and Mathematical Physics, Academic Press, New York, London, Tokyo, 1986.

[13] Albeverio, S., Gesztesy, F., Høegh-Krohn, R. and Holden, H., Solvable Models in Quantum Mechanics, Springer-Verlag, New York, 1988.

[14] Pavlov, B.S., The theory of extensions and explicitly-solvable models, Uspechi Mat. Nauk. 42, No 6 (1987), 99-131 (in Russian); English transl. in Russian Math. Surveys 42, No 6 (1987).

[15] Pavlov, B.S. and Popov, I.Yu., Acoustic model of zero-width slits and hydrodynamic stability of boundary layer, Teor. Mat. Fiz. 86, No 3 (1991), 391-401 (in Russian); Engl. transl. in: Theor. Math. Phys. (USA) 86 (1991).

[16] Popov, I.Yu., The extension theory and diffraction problems, Lect. Notes in Physics 324 (1989), 218-229.

[17] Popov, I.Yu., The stability of boundary layer and the model of suction through a small opening, in book: "Schrödinger Operators, Standard and Non-standard" (World Scientific, Singapore, New Jersey, London, Hong Kong, 1989) 403-404.

[18] Popov, I.Yu., A model of zero-width slits and the real diffraction problem, Operator Theory: Advances and Applications. Birkhäuser Verlag Basel 46 (1990), 195-196.

[19] Popov, I.Yu., The extensions theory and the opening in semitransparent surface, J. Math. Phys. 33 (1992), 1685-1689.

[20] Popov, I.Yu., The resonator with narrow slit and the model based on the operator extensions theory, J. Math. Phys. 33 (1992), 3794-3801.

[21] Moffatt, H.K., Viscous and resistive addies near a sharp corner, J. Fluid Mech. 18 (1964), 1-18.

[22] Karpeshina, Yu.E. and Pavlov, B.S., Zero-range interaction for a biharmonic and poliharmonic equations, Mat. Zametki. 40, No 1 (1986), 49-59 (in Russian). 
[23] Proudman, I. and Pearson, J.R.A., Expansions at small Reynolds number for the flow past a sphere and a circular cylinder, J. Fluid. Mech. 2 (1957), 237-262.

[24] Berezin, F.A., On the Lee model, Mat. Sbornik. 60 No 4 (1963) 425-446. (In Russian).

[25] Bognar, J., Indefinite Inner Product Spaces, Springer-Verlag, Berlin, 1974.

[26] Buchwald, V.T., Eigenfunctions of plane elastostatics. 1. The strip, Proc. Royal Soc. London. Ser. A 277, No 1370 (1964), 385-400. 